\title{
EDUCAÇÃO EM ENGENHARIA: A TECNOLOGIA COMO ALIADA NO PROCESSO DE DEMOCRATIZAÇÃO DO CONHECIMENTO CIENTÍFICO E TECNOLÓGICO EM MEIO A PANDEMIA
}

DOI: $10.37702 / 2175-957 X . C O B E N G E .2021 .3604$

Lívia Firmani Silva - liviafirmanis@outlook.com

Universidade Tecnológica Federal do Paraná

Rua Manoel Palma Cano 558

86150-000 - Alvorada do Sul - PR

Emilli Fernanda Cruz de Oliveira - emillioliveira@alunos.utfpr.edu.br

Universidade Tecnológica Federl do Paraná UTFPR

Avenida José Custódio de Oliveira 1206

87300-020 - Campo Mourão - PR

Elis Regina Duarte - elisdu@yahoo.com.br

Universidade Tecnológica Federal do Paraná

Rua Doutor Washington Subtil Chueire 330

84017-220 - Ponta Grossa - PR

Karla Silva - karla@utfpr.edu.br

Universidade Tecnológica Federal do Paraná

R. Rosalina Maria Ferreira, 12331233

87301-899 - Campo Mourão - PR

Tanatiana Ferreira Guelbert - tguelbert@gmail.com

Universidade Tecnológica Federal do Paraná

Rua das Garças 233

87300-240 - Campo Mourão - PR

Resumo: Este artigo tem por objetivo abordar como o Ensino à Distância aliado à tecnologia podem torna-se ferramentas ponderosas e imprescindíveis, vislumbrando uma alternativa significativa, para contribuir com o processo de aprendizagem na área da educação em engenharia. Acompanhando a realidade pandêmica e a vivência sofrida por toda a população mundial, nota-se a transformação educacional em todos os setores, baseada no uso integral da 
internet. Pensando em aproximar toda a comunidade, acadêmicas e professoras da Universidade Tecnológica Federal do Paraná, integrantes do projeto de extensão Oxys Azeotetic, realizaram visitas técnicas virtuais à empresas, com alcance à toda comunidade interna e externa da universidade. Por meio das redes sociais para divulgação e transmissão das lives, as visitas virtuais proporcionaram democratização do conhecimento, de maneira lúdica, prática e científica, a todos os espectadores. Tal ação foi encarregada por mostrar a veracidade em diversos setores da Engenharia Química e correlatas, possibilitando aproximar este universo de pessoas em diferentes níveis de formação, tais como alunos de Ensino fundamental e médio, universitários, professores e profissionais formados. Foi possível constatar por meio dos depoimentos, que esta iniciativa gerou um despertar, nos espectadores, por um interesse em áreas de engenharia, bem como auxiliando em suas decisões futuras aos acadêmicos a fim de suas especializações, além de divulgar as áreas de atuação do engenheiro.

Palavras-chave: Educação em Engenharia. Pandemia. Visitas Técnicas Virtuais. Ensino a Distância. Tecnologia. 


\section{EDUCAÇÃO EM ENGENHARIA: A TECNOLOGIA COMO ALIADA NO PROCESSO DE DEMOCRATIZAÇÃO DO CONHECIMENTO CIENTÍFICO E TECNOLÓGICO EM MEIO A PANDEMIA}

\section{INTRODUÇÃO}

A pandemia trouxe diversos desafios a serem enfrentados pela população mundial, sendo exemplos, a crise econômica, sanitária, na área da saúde e política, além dos desafios pessoais e educacionais. A enorme diversidade de realidades educacionais, sociais e econômicas dentro do país mostra-se como um grande desafio mesmo em períodos não emergenciais. Isso implicou, com urgência, na revisão e adequação do modelo de educação utilizando a tecnologia como suporte através de novos formatos que tornassem o acesso à educação ainda mais público. Tendo em vista que o cenário atual é ainda mais desafiador, é necessário que haja novas formas de democratizar o conhecimento e mapear ações para curto e médio prazo (GRANDISOLI, 2020).

Reinventar-se e adequar-se ao que o mundo está vivenciando é um fator decisivo na atualidade. A convivência pessoal passou a ser substituída pela virtual, assim como todas as ações coletivas, as quais envolviam um conjunto de pessoas. Sendo assim, novos métodos foram criados para tornar a convivência e relação entre as pessoas algo possível, a internet mostra-se então como uma poderosa aliada, possibilitando uma infinidade de ações (OLIVEIRA, 2020).

Por meio da internet barreiras foram quebradas, a comunicação instantânea passou a ser comum, o contato virtual entre pessoas a longas distâncias tornou-se algo rotineiro, além de ser através deste meio de navegação o possível encontro de informações sobre a pandemia e formas de proteção. Pode-se observar que existem diversas alternativas ao alcance dos indivíduos, basta tornar o acesso a essas ferramentas mais acessíveis e com isso, aproveitar os benefícios trazidos pelo novo cenário.

Objetivando democratizar o ensino nas mais diversas áreas do conhecimento, tornando-as fáceis de serem visualizadas e absorvidas pela população, o time Oxys Azeotetic, fruto de um projeto de extensão realizado pela Universidade Tecnológica Federal do Paraná - UTFPR - Campus Campo Mourão, composta por sete alunas do curso de Engenharia Química e coordenada por três professoras, realizou Visitas Técnicas Virtuais, com o intuito de mostrar a atuação dos(as) profissionais de engenharia em diversas áreas de atuação. Através dessa proposta inovadora, áreas aplicadas na Engenharia Química e correlatas, foram apresentadas: a energia proveniente do Observatório Nuclear, o funcionamento interno da Farmattiva, uma farmácia de manipulação e as práticas internas da produção eletrônica da Uartronica, empresa localizada em Portugal.

A apresentação dessas visitas técnicas alcançou e integrou não apenas o público universitário, mas também alunos do ensino médio, profissionais das áreas relacionadas e docentes, isto é, uniu a universidade com a comunidade externa. Com as transmissões das visitas foi possível, também, conhecer, por meio das telas e das redes sociais, como uma indústria funciona, os processos envolvidos em sua produção, a rede de distribuição até chegar ao consumo, podendo este ser um medicamento, um produto eletrônico ou até mesmo a energia elétrica.

Ter acesso aos detalhes das áreas correlatas às engenharias oportunizou um despertar de interesse naqueles que estão no ensino médio ou que ainda não escolheram uma profissão a ser seguida. E essa iniciativa pode ainda levar este público a se 
encantarem pela engenharia e assim decidirem seguir este caminho e, futuramente, a área de atuação com maior afinidade e interesse.

\section{2 \\ OPORTUNIDADES DO ENSINO À DISTÂNCIA EM MEIO A PANDEMIA DO} COVID-19

A partir dos anos de 1996, com a regulamentação que tornou válida a educação à distância (EAD) para tosos os níveis de escolarização, por meio da Lei n. ${ }^{\circ}$ 9.394, esta modalidade de ensino começou a tomar espaço nas instituições no Brasil (BRASIL, 1996). Desde então, a educação a distância vem se mostrando como um grande contribuinte para modificações tecnológicas no setor de educação e treinamento, essa modalidade de ensino torna o acesso ao conhecimento e a certificação profissional de certa forma mais acessível ao público (LITTO, 2010). Poder adquirir conhecimentos sem precisar ter contato direto com outros indivíduos foi um fator extremamente relevante perante a atual situação de pandemia.

Uma série de estudos a respeito dos tipos de aprendizagem foram realizados em paralelo, com o objetivo de aperfeiçoar essa metodologia de ensino. Dentre os principais tipos de aprendizagem, destaca-se a espacial, que está associada à percepção visual, onde os estímulos visuais são fundamentais para a absorção dos conteúdos. Pensando nisso, um dos fatores que contribuem com a utilização de mídias nos sistemas lineares de organização da informação é a conquista da atenção do espectador através de imagens, cores e pessoas, elementos que interligados possibilitam uma melhor associação de contexto e fixam melhor as informações (PASSARELLI, 2003).

Estabelecendo a educação a distância como a melhor alternativa para a educação em meio a pandemia, passa a ser reconhecida a independência de um professor para o aprendizado direto e da presença simultânea deste e seus alunos. Tecnologias precisam e são incrementadas renovando todo o ensino, contribuindo para uma formação consolidada, pois tendo conhecimento de todos os recursos a serem usados, cabe ao discente buscar conteúdos e meios satisfatórios para a construção de sua cognição, alcançando destaque suficiente em tempos de quarentena.

Neste sentido, o impacto causado pela pandemia nos setores educacionais, remete a discussão sobre a importância da educação a distância como alternativa. Sabe-se que a tecnologia está ligada diretamente à evolução digital, a qual pode permitir experiências proveitosas na educação. Do exposto, retomando o cenário atual do mundo, que se encontra em uma realidade conturbada perante uma pandemia ocasionada pelo vírus identificado como COVID-19, que tornou o isolamento social uma medida de prevenção e diversos setores foram afetados, destacando o educacional, em que os estudantes ficaram impedidos de ir às instituições de ensino (CORDEIRO, 2020).

As instituições educacionais tiveram de se empenhar na busca de novas modalidades de ensino, tendo como auxílio às tecnologias digitais, a internet. A adaptação a essa nova realidade se mostrou com certa dificuldade no início, mas docentes e discentes decidiram usar criatividade e organização em suas rotinas para dar continuidade às atividades escolares, usando recursos digitais como suporte. Reaprender a ensinar e reaprender a aprender são processos contínuos na educação com isolamento social no país (CORDEIRO, 2020).

Neste contexto, universitários buscaram por recursos que pudessem ser usados em meios digitais, e que estes, permitissem uma melhor familiarização com a tecnologia por parte do público. Assim, palestras e visitas técnicas virtuais se mostraram como um ensino midiático que prenderia a atenção dos espectadores, com diálogos e recursos visuais de 
temáticas de interesse, uma oportunidade para o público interessado adquirir maiores conhecimentos a respeito de uma diversidade de temas, ultrapassando barreiras de distância, protocolos de segurança e fortalecendo os métodos de comunicação.

Pensadores contemporâneos afirmam, baseando-se nos eventos vivenciados, que a educação não será mais a mesma e que a necessidade de implementar novas alternativas nos métodos de ensino é real. As aulas do modo tradicional, devem ser auxiliadas com recursos tecnológicos, de modo a facilitar a fixação de conteúdos e uma maior diversidade de informações. O mundo mudou em sua dinâmica, assim como o âmbito educacional de maneira inopinada diante dos desafios impostos pela Covid-19.

\section{METODOLOGIA}

O presente estudo se caracteriza como aplicado na medida em que foi desenvolvido de forma prática, com a implantação de uma modalidade inovadora de visitas técnicas virtuais com interação em tempo real do público-alvo. É uma pesquisa qualitativa, pois não tem a intenção de evidenciar dados estatísticos e nem traduzir em números os resultados alcançados, por este motivo também se classifica como exploratória e descritiva, evidenciando que o estudo analise os exemplos para estimular a compreensão (GIL, 2008) e descreve fatos e fenômenos reais (TRIVINOS, 1987).

A ideia inicial da realização remota das visitas técnicas, pela equipe do projeto de extensão da Universidade Tecnológica Federal do Paraná (UTFPR), denominado de Oxys Azeotetic, surgiu espelhando-se no CADEQ - Centro Acadêmico de Engenharia Química da UTFPR - CM, o qual havia elaborado uma iniciativa nestes moldes em que, partes das integrantes, fazem parte de ambos (CADEQ e Oxys Azeotetic). Com base na experiência vivenciada, a equipe decidiu buscar por temáticas inéditas, como uma forma de levar ao público o conhecimento de áreas diferentes das mais comuns. Pensando em inovar e evidenciar, principalmente, aos universitários, para se familiarizarem com a rotina, os processos e os trabalhos de uma indústria.

As visitas virtuais aconteceram por meio do trabalho em equipe da Oxys Azeotetic, cujas responsabilidades foram divididas entre as integrantes, as quais entraram em contato com as empresas e palestrantes que se tornaram parceiros do projeto, e assim puderam agendar a melhor data para realizar os eventos no formato online. É importante salientar o planejamento, organização e as preparações para que as transmissões ocorressem corretamente, minimizando possíveis problemas, bem como o reforço de parceiros para a filmagem.

Após a confirmação, a primeira ação foi desenvolver o material de divulgação, realizado totalmente pelas integrantes, em seguida foram feitas divulgações através da conta do Instagram e Facebook do projeto, com a publicação de imagens informativas do evento e em seguida o convite foi compartilhado, por e-mail, entre os universitários, professores, servidores, colegas e escolas do ensino médio, estendendo-se a pessoas que estariam interessadas em assistir a visita técnica. Também foram utilizadas as contas pessoais das redes sociais das integrantes do projeto, incorporando inclusive o Linkedln para a divulgação.

Mesmo não sendo uma ação presencial para a maioria, sem a experiência de conhecer lugares e indústrias, o diferencial em realizar uma transmissão é marcado, principalmente, pelo livre acesso, pois trata-se de um ensejo a todos aqueles que buscam por tal conhecimento, além da filmagem estar disponível indeterminadamente no canal do Youtube do projeto de extensão da Oxys Azeotetic, podendo ser revista e avaliada de acordo com a necessidade. 
A participação do público foi comprovada por meio do preenchimento de um breve questionário, possibilitando a emissão de declarações de participação aos participantes, contabilizada com uma hora complementar por visita.

De notória importância, a interação através do chat com os espectadores possibilitou o direcionamento de dúvidas e questionamentos deixadas pelos mesmo durante a realização da visita virtual ao palestrante, o que agregou para uma melhor compreensão e aprendizado das visitas realizadas, promovendo uma interação em tempo real. Além de obter-se conhecimento da localidade, instituição e do curso daqueles que assistiam.

\section{RESULTADOS E DISCUSSÕES}

Organizar e realizar as visitas técnicas virtuais trouxe experiências importantes para o time, marcada pela responsabilidade em ter um contato direto com o público, sendo alunos de outras universidades, professores, colegas e a comunidade externa. Além de todo o conhecimento científico adquirido, a equipe também teve a oportunidade de aprimorar soft skills relacionadas com a organização, trabalho em equipe, resolução de problemas, negociação, gestão de tempo e flexibilidade.

Após cada live, realizada por meio da plataforma de transmissão do Youtube, os resultados foram contabilizados e se apresentaram como positivos, a presença de depoimentos com elogios e agradecimentos foram substanciais, assim como possibilidades de parcerias educacionais.

A primeira visita virtual foi realizada no dia 22 de outubro de 2020 , na qual os visitantes tiveram acesso aos detalhes do Observatório Nuclear em Angra dos Reis - Rio de Janeiro (Figura 1). A apresentação foi realizada durante a Semana da Ciência Nuclear, sendo ministrada, inicialmente, por uma engenheira nuclear e tendo continuidade pelo supervisor de comunicação da Eletronuclear, responsável por explicar o funcionamento da engenharia nuclear e dessa singular usina. A visita, assim como as demais, estão disponíveis no canal da Oxys Azeotetic no Youtube e pode ser acessada a qualquer momento (OXYS AZEOTEIC, 2020).

Figura 1 - Visita Técnica Virtual ao Observatório Nuclear de Angra dos Reis.

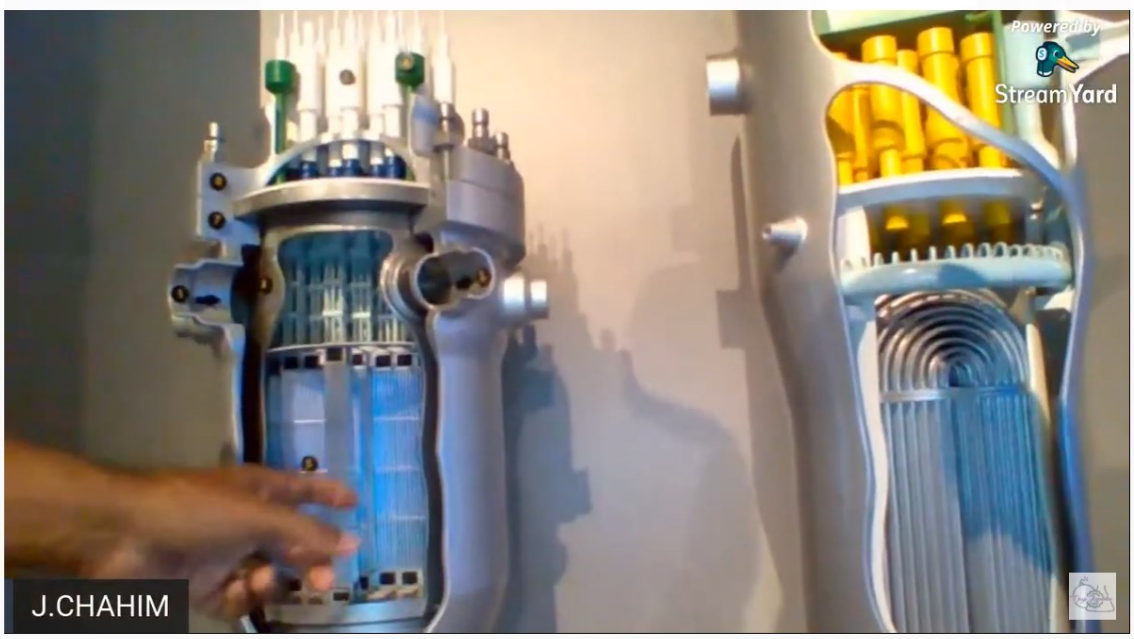

Visita Técnica Virtual ao observatório Nuclear em Angra dos Reis. 
Foi obtido um alcance de mais de duas mil e duzentas visualizações, como pode ser observado na Figura 1. Com a interação de universitários dos 13 câmpus da UTFPR, UFES (Universidade Federal do Espírito Santo) e alunos do Colégio Salesiano Dom Bosco de Americana - São Paulo, além de toda a comunidade externa.

Alguns dos comentários recebidos e registrados na live foram: "Muito boa iniciativa! Muito interessante esse assunto, deu para ter uma boa ideia de como funciona uma usina, agradeço" e "Gostei muito do evento! Eu não teria a possibilidade de participar presencialmente. Espero que este formato seja utilizado mais vezes em outras visitas." (OXYS AZEOTETIC, 2020).

A segunda visita técnica virtual foi realizada diretamente de Portugal, no dia 26 de novembro de 2020, mostrando detalhes da empresa, fabricante e fornecedora de componentes eletrônicos, Uartronica. Uma das orientadoras do projeto esteve presente junto a equipe da empresa, auxiliando e dando suporte.

Através dessa visita foi possível identificar diferentes áreas de atuação dentro de uma única indústria, assim como a importância da multidisciplinaridade em que diferentes profissionais devem trabalhar em conjunto para a obtenção do produto final. Foi possível verificar também a possibilidade de empregabilidade para estudantes e egressos de universidades brasileiras que possuem interesse no campo da engenharia eletrônica e afins, pode-se destacar que a mobilidade internacional é uma oportunidade que muitos buscam para adquirir maiores experiências pessoais e profissionais (OXYS AZEOTETIC, 2021).

Figura 2 - Visita Técnica Virtual à Uartronica.

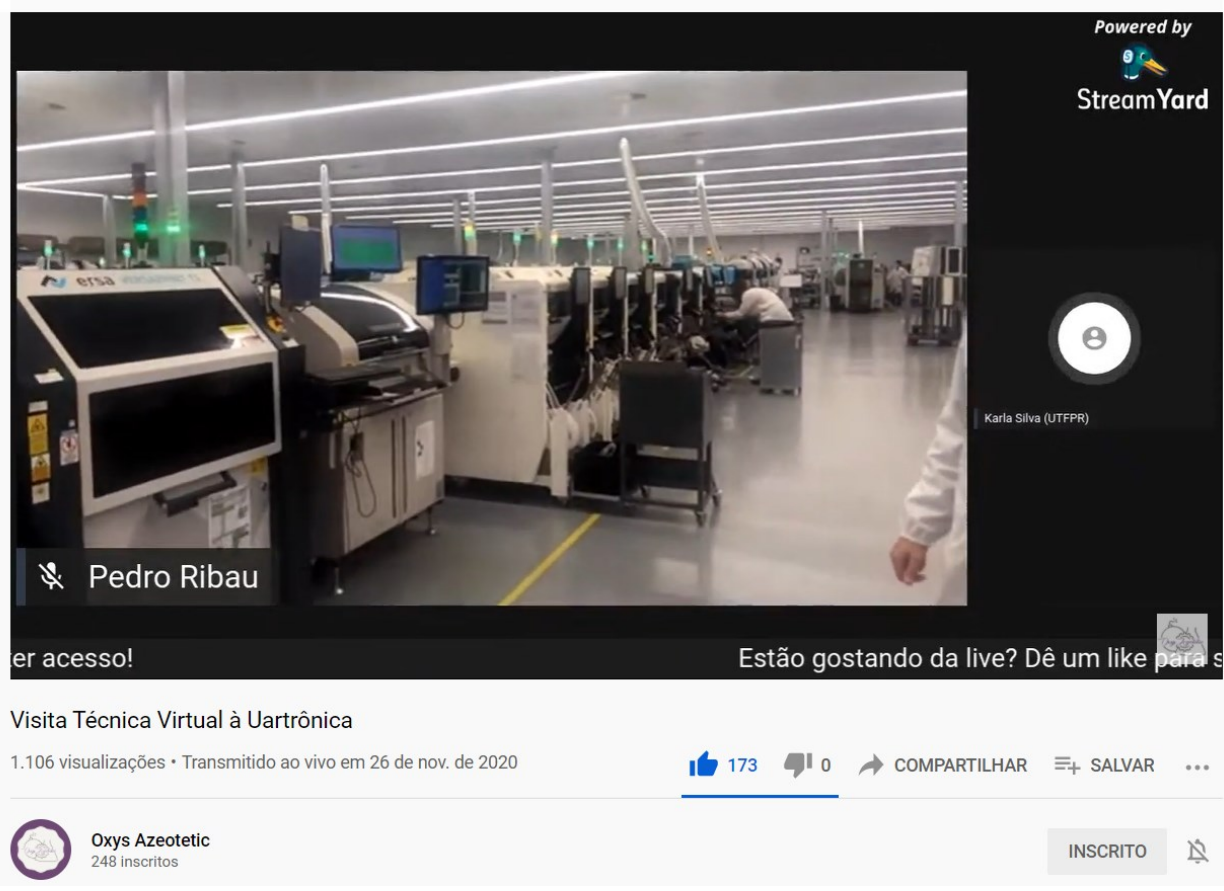

Fonte: OXYS AZEOTETIC, 2021.

Como observa-se na Figura 2, os palestrantes mostraram também como a indústria vem avançando exponencialmente nas últimas décadas utilizando as tecnologias em seu auxílio. A indústria 4.0, conceito amplamente utilizado para se referir a tecnologias voltadas para automação e troca de dados em sistemas, se mostra presente mais uma vez na sociedade, trazendo a melhoria da eficiência e produtividade dos processos. Neste sentido, 
popularizar e democratizar esse conhecimento por meio da informação é de substancial importância para o público-alvo do projeto.

A terceira e última visita realizada foi na Farmattiva, farmácia de manipulação homeopatia e produtos naturais, localizada na cidade de Goioerê, no estado do Paraná. A transmissão contou com a presença da proprietária e farmacêutica responsável pelo estabelecimento e uma acadêmica de Engenharia Química da UTFPR, membro do time Oxys Azeotetic, além do suporte de filmagem e funcionários do local.

A visita contribuiu para que o público pudesse visualizar a atuação dos engenheiros químicos, químicos, farmacêuticos e demais profissionais no setor de medicamentos. Foi possível observar também como são os equipamentos utilizados, quais são as medidas de segurança adotadas, como ocorre a manipulação de medicamentos e cosméticos, essa última parte contando ainda com a demonstração prática da fabricação de um creme dermatológico com hidroviton, visualizado na Figura 3 (OXYS AZEOTETIC, 2021).

Figura 3 - Visita Técnica Virtual à Farmattiva
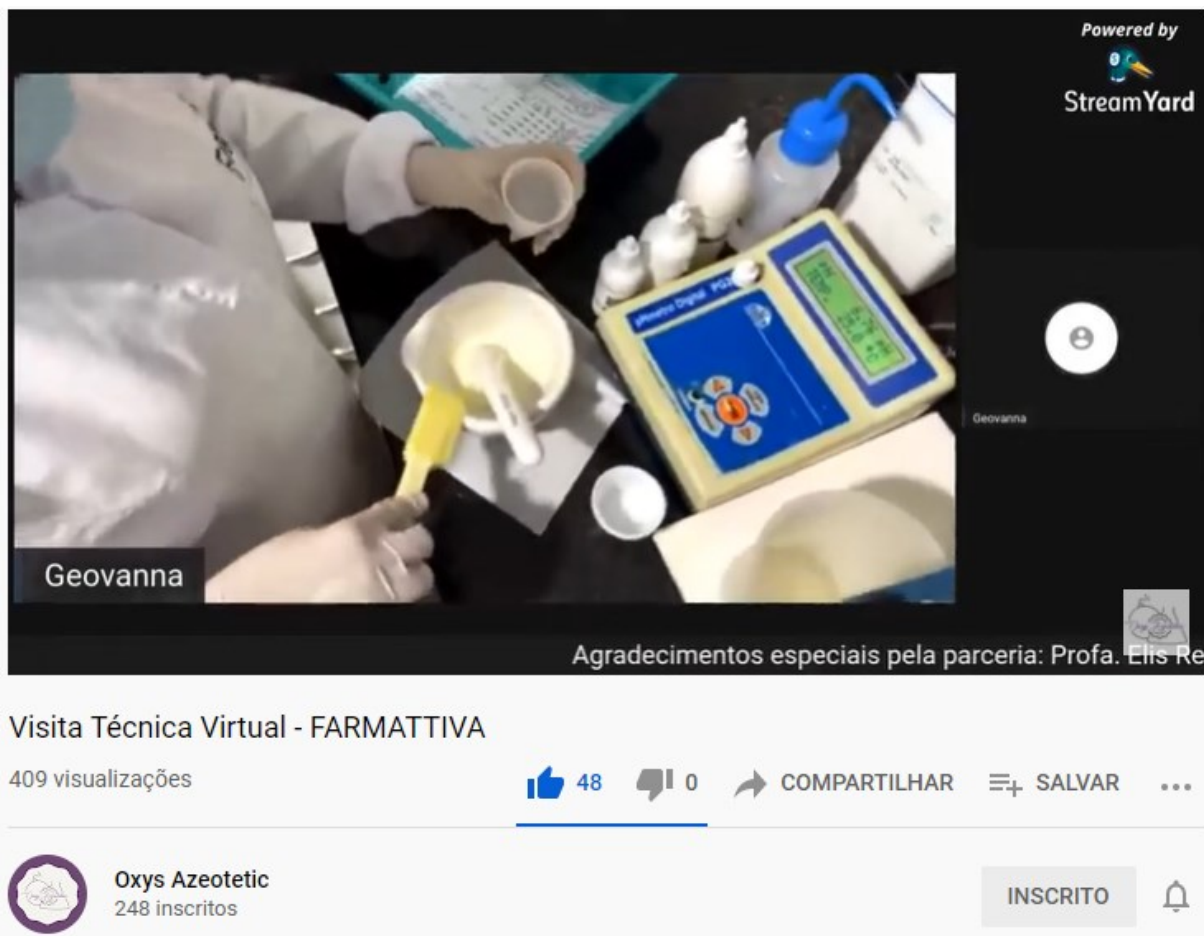

Fonte: OXYS AZEOTETIC, 2021.

É importante ressaltar que como todas as visitas foram realizadas durante a pandemia, todas seguiram os protocolos de segurança, respeitando o distanciamento entre os participantes, também fazendo a utilização de máscaras de segurança, além de ser realizado uma higienização do ambiente em que estavam localizados.

\section{CONSIDERAÇÕES FINAIS}

O cenário atual trouxe diversos desafios aos indivíduos, destacando-se aqui o âmbito educacional. Foi possível verificar que a utilização de tecnologias como aliadas no processo de ensino e aprendizagem é um fator imprescindível tratando-se do contexto vivenciado. $\mathrm{O}$ uso adequado da tecnologia na educação, em diversos casos, permite que ocorra um 
impulsionamento da aprendizagem dos alunos, portanto, se usada como uma aliada, a tecnologia fornece uma democratização do conhecimento.

Através das visitas técnicas realizadas, foi possível concluir que um ensino midiático pode ser um chamariz para o público, que se mostra interessado por possuir a oportunidade de conhecer diversas áreas do conhecimento, assim como muitas profissões e indústrias, as quais em um cenário diferente, não poderiam ser realizadas de forma presencial. Os métodos de comunicação fornecidos pelos avanços tecnológicos, devem ser cada vez mais fortalecidos, pois estes permitem que haja uma interação entre os mais diversos públicos.

Portanto, sabe-se que o mundo se mostra cada vez mais atento e que exige um desenvolvimento de competências e conhecimentos específicos de cada indivíduo. Assim, alternativas como a inserção de recursos visuais na área da educação, com o auxílio de tecnologias, devem ser incorporado no decorrer do tempo às modalidades de ensino, neste contexto voltadas para as áreas das engenharias, para assim possibilitar um fornecimento maior e mais didático de conhecimento e informação.

Esta iniciativa, das visitas técnicas virtuais, terá continuidade em função dos resultados positivos alcançados e pode ser uma alternativa replicada em outras instituições de ensino, inclusive por meio de parcerias com o projeto da Oxys Azeotetic, a qual já possui Know How para seu desenvolvimento. As parcerias, a exemplo das que foram desenvolvidas e resultaram neste artigo, corroboram o pensamento de que "sozinhos podemos chegar rápido, entretanto em times com certeza chegaremos mais longe", neste sentido é importante ressaltar que é necessário "pensar grande, começar pequeno, mas andar rápido e com os parceiros". Esta é a lição aprendida pelas integrantes da Oxys Azeotetic com o desenvolvimento do projeto.

\section{Agradecimentos}

As indústrias parceiras e à Universidade Tecnológica Federal do Paraná - Campo Mourão, por propiciar e apoiar a realização deste projeto.

\section{REFERÊNCIAS}

BRASIL. Lei № 9.394, de 20 de dezembro de 1996. Disponível em < http://www.planalto.gov.br/ccivil_03/leis/19394.htm>. Acesso em 06 mai. 2021.

\section{CORDEIRO, K. M. A. O Impacto da Pandemia na Educação: A Utilização da}

Tecnologia como Ferramenta de Ensino. 2020. Disponível em: http://repositorio.idaam.edu.br/jspui/bitstream/prefix/1157/1/O\%20IMPACTO\%20DA\%20P ANDEMIA\%20NA\%20EDUCA \%c3\%87\%c3\%830\%20A\%20UTILIZA\%c3\%87\%c3\%830\% 20DA\%20TECNOLOGIA\%20COMO\%20FERRAMENTA\%20DE\%20ENSINO.pdf. Acesso em: 1 maio. 2021.

GIL, Antônio, Carlos. Métodos e Técnicas de Pesquisa Social. 6. ed. p.57 - São Paulo: Atlas, 2008.

GRANDISOLI, E.; JACOBI, P. R.; MARCHINI, S. Educação e pandemia: desafios e perspectivas. Jornal da USP São Paulo, v. 12, 2020. Disponível em: <https://jornal.usp.br/artigos/educacao-e-pandemia-desafios-e-perspectivas/>. Acesso em: 30 abril. 2021. 
LITTO, Fredric M. Aprendizagem a distância. Imprensa Oficial do Estado de São Paulo, 2010.

OLIVEIRA, E.D. et al. A educação a distância (EaD) e os novos caminhos da educação após a pandemia ocasionada pela Covid-19. Brazilian Journal of Development, v. 6, n. 7, p. 52860-52867, 2020. Disponível em:

https://www.brazilianjournals.com/index.php/BRJD/article/view/14095/11787. Acesso em: 01 maio. 2021.

OXYS AZEOTETIC. Visita Técnica Virtual ao observatório Nuclear em Angra dos Reis. YouTube, 23 out. 2020. Disponível em:

https://www.youtube.com/watch?v=x90490aOGy0\&t=7s. Acesso em: 1 maio. 2021.

OXYS AZEOTETIC. Visita Técnica Virtual à Uartronica. YouTube, 26 nov. 2020.

Disponível em: https://www.youtube.com/watch?v=sBjOJOdivZU\&t=14s. Acesso em: 1 maio. 2021.

OXYS AZEOTETIC. Visita Técnica Virtual - FARMATTIVA. YouTube, 24 fev. 2021. Disponível em: https://www.youtube.com/watch?v=1e4SQVrVHP0. Acesso em: 1 maio. 2021.

PASSARELLI, B. Teoria das Múltiplas Inteligências aliada à Multimídia na Educação: Novos Rumos Para o Conhecimento. Escola do Futuro/USP, 2003. Disponível em: http://penta3.ufrgs.br/twiki.novo/pub/Teleducacao2008/CoAutoria/multiplasintelig.pdf. Acesso em: 1 maio. 2021.

TRIVIÑOS, A. N. S. Introdução à Pesquisa em Ciências Sociais: A Pesquisa Qualitativa em Educação. São Paulo: Atlas, 1987.

\title{
ENGINEERING EDUCATION: TECHNOLOGY AS AN ALLY IN THE PROCESS OF DEMOCRATIZING SCIENTIFIC AND TECHNOLOGICAL KNOWLEDGE AMONG PANDEMIA
}

\begin{abstract}
This article aims to address how Distance Learning combined with technology can become powerful and indispensable tools, envisioning a significant alternative, to contribute to the learning process in the field of engineering education. Following the pandemic reality and the experience suffered by the entire world population, there is an educational transformation in all sectors, based on the full use of the internet. Thinking of bringing the entire community closer together, academics and professors from the Federal Technological University of Paraná, members of the Oxys Azeotetic extension project, made virtual technical visits to companies, reaching the entire internal and external community of the university. Through social networks for the dissemination and transmission of lives, virtual visits provided democratization of knowledge, in a playful, practical and scientific way, to all viewers. This action was responsible for showing the veracity in several sectors of Chemical Engineering and related ones, making it possible to bring this universe of people together at different levels of education, such as elementary and high school students, university students, teachers and trained professionals. It was possible to verify through the testimonies, that this initiative generated an awakening, in the spectators, by an interest in
\end{abstract}


engineering areas, as well as helping in their future decisions to the academics in order to their specializations, besides divulging the areas of performance of the engineer.

Keywords: Engineering Education. Pandemic. Virtual Technical Visits. Distance learning. Technology. 